\title{
Production and qualification of an electrospun ceramic nanofiber material as a candidate future high power target
}

\author{
Sujit Bidhar, ${ }^{1}$ Valerie Goss $\odot,{ }^{2}$ Wei-Ying Chen, ${ }^{3}$ Andrei Stanishevsky, ${ }^{6}$ Meimei Li, ${ }^{3}$ \\ Slava Kuksenko, ${ }^{4}$ Marco Calviani $\odot,{ }^{5}$ and Robert Zwaska ${ }^{1}$ \\ ${ }^{1}$ Fermi National Accelerator Laboratory, Batavia, Illinois 60510, USA \\ ${ }^{2}$ Chicago State University, 9501 S. King Drive Chicago, Illinois 60628, USA \\ ${ }^{3}$ Argonne National Laboratory, 9700 S. Cass Avenue, Lemont, Illinois 60439, USA \\ ${ }^{4}$ UK Atomic Energy Authority Culham Science Centre, Abingdon, \\ Oxfordshire OX14 3DB, United Kingdom \\ ${ }^{5}$ CERN, Geneva CH1211, Switzerland \\ ${ }^{6}$ University of Alabama at Birmingham, Alabama 35294, USA
}

(Received 27 January 2021; revised 26 July 2021; accepted 18 November 2021; published 7 December 2021)

\begin{abstract}
In an effort to develop and design next generation high power target materials for particle physics research, the possibility of fabricating nonwoven metallic or ceramic nanofibers by electrospinning process is explored. A low-cost electrospinning unit is set up for in-house production of various ceramic nanofibers. Yttria-stabilized zirconia nanofibers are successfully fabricated by electrospinning a mixture of zirconium carbonate with high-molecular weight polyvinylpyrrolidone polymer solution. Some of the inherent weaknesses of electrospinning process like thickness of nanofiber mat and slow production rate are overcome by modifying certain parts of electrospinning system and their arrangements to get thicker nanofiber mats of millimeter order at a faster rate. Continuous long nanofibers of about hundred nanometers in diameter are produced and subsequently heat treated to get rid of polymer and allow crystallize zirconia. Specimens were prepared to meet certain minimum physical properties such as thickness, structural integrity, thermal stability, and flexibility. An easy innovative technique based on atomic force microscopy was employed for evaluating mechanical properties of single nanofiber, which were found to be comparable to bulk zirconia. Nanofibers were tested for their high-temperature resistance using an electron beam. It showed resistance to radiation damage when irradiated with $1 \mathrm{MeV} \mathrm{Kr}^{2++}$ ion. Some zirconia nanofibers were also tested under high-intensity pulsed proton beam and maintained their structural integrity. This study shows for the first time that a ceramic nanofiber has been tested under different beams and irradiation condition to qualify their physical properties for practical use as accelerator targets. Advantages and challenges of such nanofibers as potential future targets over bulk material targets are discussed.
\end{abstract}

DOI: 10.1103/PhysRevAccelBeams.24.123001

\section{INRODUCTION}

In high-energy particle physics research, there is a growing demand of increased sub-atomic particle production in order to understand the building blocks of universe. This could be achieved by increasing the proton beam power in a particle collider to hit a target material. Particle accelerators in future are expected to operate at multi-MW beam power for advancing research in high-energy particle physics. For instance, some facilities in near future are planning to use multi-MW beam power for delivering highintensity neutrino beams. Spallation sources elsewhere, at

Published by the American Physical Society under the terms of the Creative Commons Attribution 4.0 International license. Further distribution of this work must maintain attribution to the author(s) and the published article's title, journal citation, and DOI. spallation neutron source and European spallation source have started building their facilities for multi-MW beams $[1,2]$. It is expected that the targets in such facilities would survive intense thermal stress cycles as well as severe radiation damage leading to swelling, transmutant, gas formation, embrittlement and change of other physical and mechanical properties. In recent years, intense pulsed proton beam of $750 \mathrm{~kW}$ has been successfully used on graphite targets to produce neutrinos [3]. Such a focused pulsed beam locally heats a small area of the target that expands rapidly during a short time giving rise to compressive stress waves, which in turn may lead to fatigue cracking. Although current solid targets have successfully performed at this beam power, their performance is uncertain in near future when the targets will be exposed to multi-MW beams. Target designs, at the moment, focus mostly on thermal issues to avoid local melting or vaporization of the target. Some facilities have started using 
rotating tungsten [4], granular tungsten target [5], tungsten powder target [6], and liquid mercury targets [7]. However, as the beam intensity increases such targets will face similar fate of fixed targets. Increasing beam power and intensity would increase the thermal fatigue stress amplitude, which would shorten the fatigue life of the target even though it operates well under yield strength, hence decreasing facility reliability, increasing downtime and cost. Liquid targets will have more complex problems of cavitation and pressure [8].

To mitigate these issues, it is proposed to design a target with sinuous microstructure instead of solid continuum. Since the diameter of nanofiber is much smaller than the beam spot size, there will be no macroscopic thermal gradient across a single nanofiber. Besides there are many gaps between the adjacent nanofibers, which would prevent any kind of compressive stress waves. High-surface area of the nanofibers and high porosity of the nanofiber mat may enable effective cooling from the interior of the mat and even from the beam center by passing helium gas through such porous target. It is also expected to offer resistance to radiation damage due to nanopolycrystalline structures with large number of grain boundaries and free surfaces that would act as sinks to irradiation induced defects. The submicron diameter and ubiquitous grain boundaries of nanofiber reduces the mean free path for low-solubility transmutation products including helium gas that formed due to proton beam interaction with material, to escape out of the material and hence prevent any kind of swelling which causes cracking in bulk material. Unlike a bulk solid material where localized damage could lead to catastrophic failure of entire target structure the nanofiber mat as a whole can maintain its integrity even if there is local damage of a few nanofibers. Although nanofiber microstructure offers above benefits, it is desired that the target made with nanofiber mat should be able to withstand extreme temperature generated due to high-energy proton beam interaction. It should satisfy certain physical parameters such as thickness, structural integrity, thermal stability, and flexibility.

It is hypothesized that if a target with nanofiber microstructure could be made out of ceramics like zirconia, it would offer higher melting point and could withstand extreme heat from beam interaction. Zirconia in bulk form is brittle and have poor thermal shock resistance. However, zirconia in nonwoven nanofiber mat form is expected to have better thermal shock resistance. In order to verify these postulated beneficial properties of nanofiber in target material, we prepared such materials and carried out subsequent micromechanical and irradiation tests on zirconia nanofibers, which are presented here.

There are several methods like melt blowing, drawing, laser spinning to produce nanofibers $[9,10]$. In recent years, electrospinning technique has been successfully used in making nanofiber of many different materials

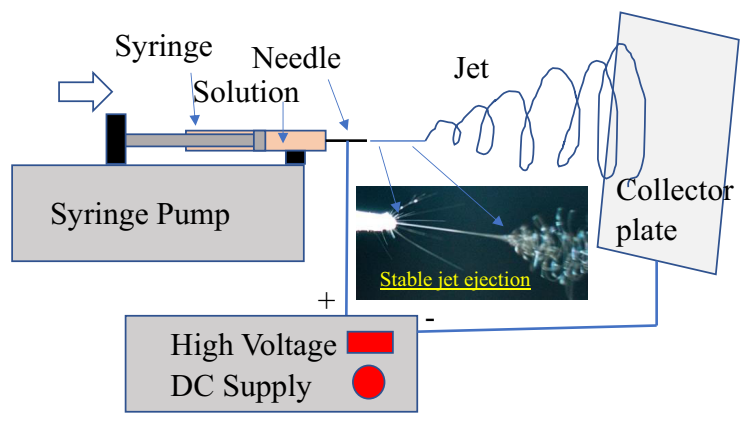

FIG. 1. Schematic of electrospinning set up.

inexpensively and imparting various functionalization [11-15]. Electrospinning method offers easy production of customized composite ceramic nanofiber with specific functional properties. Recently ceramic nanofibers have found application in radiation shielding in spacecraft, sensors and electronic of nuclear industries, as well as structural components [16-18]. Figure 1 shows a basic electrospinning setup where a syringe with a blunt needle containing high-molecular weight polymer solution is slowly pushed by a plunger. A high potential is applied to the needle while a metal collector plate is kept in front of the syringe at certain distance and at ground potential to maintain a high-electric field. As the solution comes out of the needle forming a droplet, the electrical charge concentration on the surface of solution droplet induces the force that overcomes the surface tension causing the droplet to stretch under the influence of an electric field and form a liquid jet that accelerates toward the collector plate. Further jet stretching and solvent evaporation leads to the formation of solid fibers with the diameter of less than one micrometer by the time it reaches the collector plate forming a nonwoven fiber mat.

Although a lot of work has been done in electrospinning of polymeric nanofibers, much less have been done on ceramic or metallic nanofibers production especially in improving their thermal, mechanical, and radiation damage resistance properties. Ceramic nanofibers are finding applications in filtration and membrane technology [19] as well as in energy storage devices like fuel cells and catalysts in catalytic converters. In these applications, the fiber mat is very thin and structural integrity of the fiber mat is not an issue as they are often sandwiched between other substrate materials. But in the present application, it is required to have stand-alone nanofiber mat as a substrate material that will undergo damage under intense pulsed beam. Hence, the nanofiber mat should to be thick enough to have structural strength on its own to sustain the thermal stresses due the high-intensity pulsed beam heating. Besides, very little work has been done in the area of physical characterization of single ceramic nanofiber.

In this study, we present the fabrication and qualification of thicker zirconia nanofiber mat using a low-cost low power output lab-scale electrospinning unit. A specific heat 
treatment process was used to get rid of polymer component of as-spun nanofibers and obtain zirconia nanofibers of 100-200 nm diameter consisting of nano-polycrystalline grains of few tens of nanometer in size. Micromechanical tests were carried out to evaluate mechanical properties of single nanofiber using atomic force microscope (AFM) while thermal response was carried out under an intense electron beam. Some of these produced nanofiber mats were also exposed to high-energy proton beam at CERN, Switzerland to test their resistance to intense thermal shock due to high-energy proton beam and qualify these materials as future high-power targets. Samples were also irradiated with low-energy $\mathrm{Kr}^{++}$ion beam to evaluate their resistance to displacement damage.

\section{MATERIALS AND METHOD}

\section{A. Electrospinning setup}

Figure 2(a) shows a modified version of a conventional electrospinning setup to get thicker nanofiber mat using a custom-made dual-polarity power supply and a negative ionizer. Figure 2(b) shows the inside of the custom-made power supply, which is made up of two pieces of highvoltage dc-dc converter, Ultravolt 40A12-P4 (Advanced energy, USA). It has two output voltage could be varied independently between 0 to $+40 \mathrm{kV} \mathrm{DC}$ and 0 to $-20 \mathrm{kV}$ $\mathrm{DC}$ in dual polarity mode. Moreover, it can be operated out of a $9 \mathrm{~V}$ cell as well as $12 \mathrm{~V}$ dc adapter. The limit on output power to $4 \mathrm{~W}$ makes it much safer to use without posing any electrical hazards.

A three-dimensional printed holder with multiple syringes was used to increase the production rate. Five 21-gauge blunt needles with nominal internal diameter of $0.6 \mathrm{~mm}$ are used in this setup. The positive terminal of the high-voltage supply was connected to the needles and was set at $17 \mathrm{kV}$ while the negative terminal was connected to a knife-edge at $5 \mathrm{kV}$. The negative potential was chosen so that the sharp edge of the knife increases the local potential causing ionization of air molecules while the distance between the knife-edge and the cylindrical collector was adjusted to prevent breakdown of air. The cylindrical collector of $76.2 \mathrm{~mm}$ diameter and $203.2 \mathrm{~mm}$ long made up of aluminum, was kept at ground potential. The distance between the collector and the syringe needles was maintained at $15 \mathrm{~cm}$ and the knife-edge which acts as negativeion generator, was placed behind the rotating drum at a distance of $1 \mathrm{~cm}$. The collector was given a fixed slow rotation of 100 RPM during electrospinning process. A syringe pump, NE-1000 (New Era pump system, USA) was used to slowly pump the solution at a rate of $0.5 \mathrm{ml} / \mathrm{h}$ per needle. Electrospinning process was continued for about $10 \mathrm{~h}$ to get a thick nanofiber mat with thickness up to $3 \mathrm{~mm}$ which was collected over the cylindrical rotary collector in the form of a nonwoven sheet. The as-spun nanofiber mat was easily peeled off from the collector drum and cut into small pieces of 1-in. squares for further heat treatment.

\section{B. Raw materials for electrospinning}

The production of ceramic nanofiber is outlined in Fig. 3. Figure 3(a) describes the successive steps starting with raw materials. Ten grams of zirconium carbonate $\left(\mathrm{CH}_{2} \mathrm{O}_{7} \mathrm{Zr}_{2}\right.$, from American Elements) was added to $10 \mathrm{~g}$ of deionized water and stirred using a magnetic stirrer for $30 \mathrm{~min}$. A white color solution was obtained to which $15 \mathrm{~g}$ of acetic acid $\left(\mathrm{CH}_{3} \mathrm{COOH}\right)$ was added slowly maintaining a constant temperature of $60^{\circ} \mathrm{C}$ and stirring for another $15 \mathrm{~min}$. A clear solution was obtained indicating complete dissolution of zirconium carbonate. Stirring was continued and solution was brought back to room temperature. One gram of yttrium nitrate hexahydrate $\left(\mathrm{Y}\left(\mathrm{NO}_{3}\right)_{3} \times 6 \mathrm{H}_{2} \mathrm{O}\right.$, SigmaAldrich USA) was added to this clear solution at room temperature as a phase stabilizer. Two grams of Polyvinyl pyrrolidone (Mw $1.3 \times 10^{6} \mathrm{~g} / \mathrm{mol}$, Sigma-Aldrich, USA) was then added to this solution stirring constantly at $60^{\circ} \mathrm{C}$ for about $30 \mathrm{~min}$ or till it dissolves completely. Then, the solution was brought back to room temperature and stirred (a)

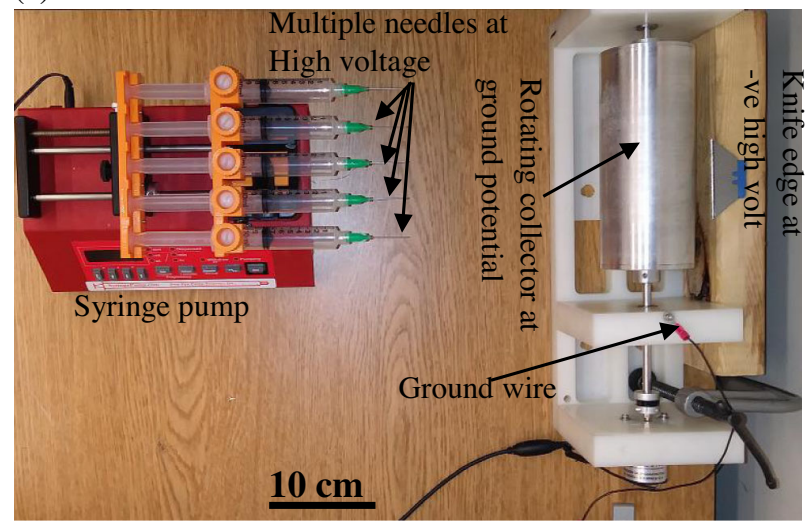

(b)

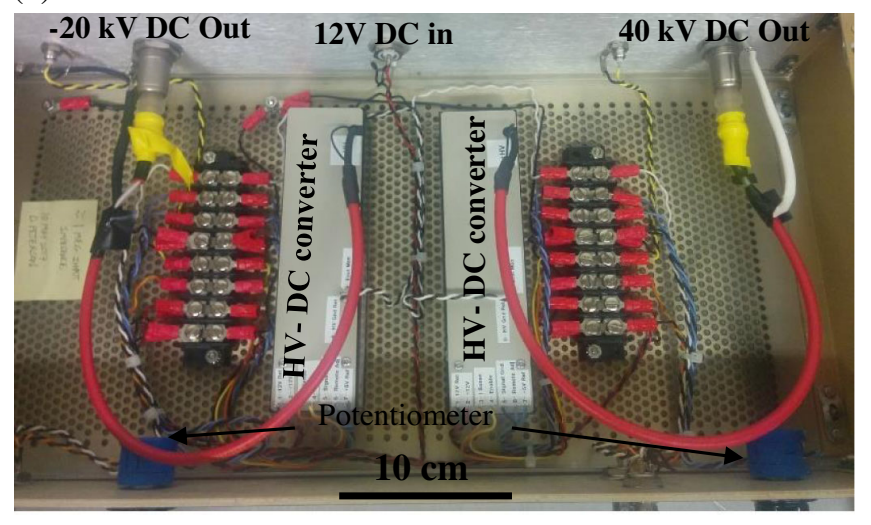

FIG. 2. Production of zirconia nanofiber mat using modified electrospinning setup. (a) electrospinning arrangement for high production rate of thick mats and (b) custom-made high- voltage power-supply. 
(a)

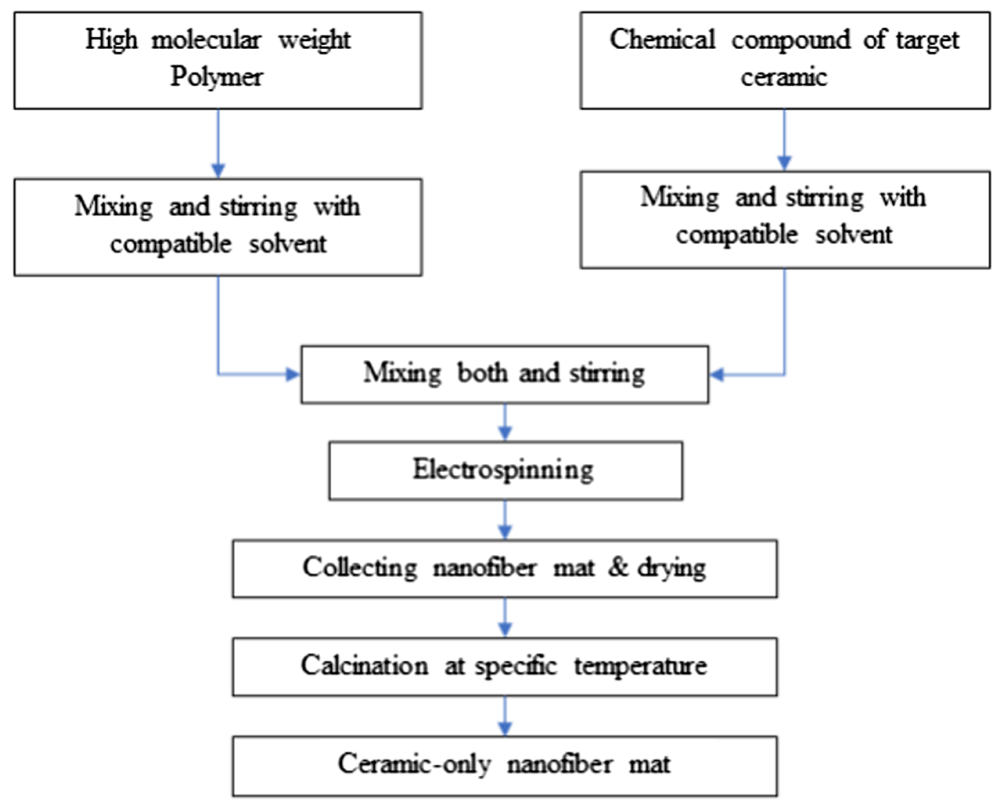

(b)

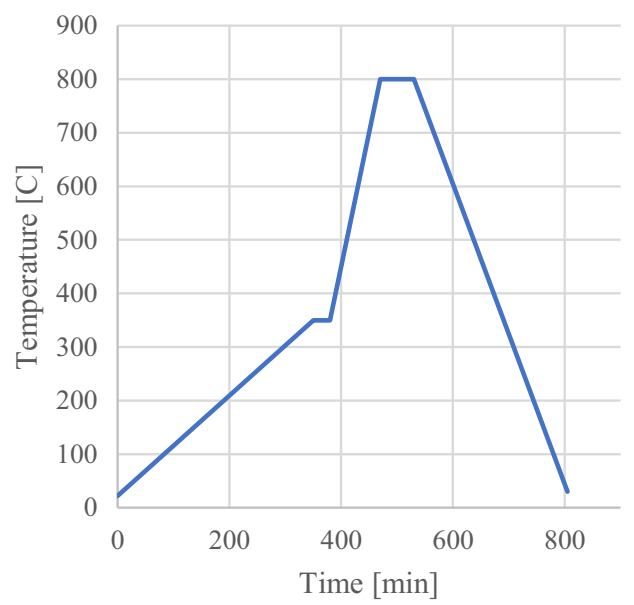

FIG. 3. Ceramic nanofiber production. (a) Process flow chart and (b) Heat treatment profile for Zirconia.

for another $6 \mathrm{~h}$ before loading it to a syringe for electrospinning.

\section{Heat-treatment of nanofiber}

Figure 3(b) shows the heat treatment protocol followed in this work for fabrication of zirconia nanofibers. The asspun nanofiber mat piece was placed in an alumina crucible and heated inside a tube furnace by flowing atmospheric air. A two-stage heating profile was applied. In the first stage, the temperature was raised from room temperature to $350{ }^{\circ} \mathrm{C}$ heating at a slow rate of $0.5^{\circ} \mathrm{C} / \mathrm{min}$ and holding the temperature for $30 \mathrm{~min}$. This allowed the polymeric components of nanofiber to decompose and subsequently vaporize leaving only the inorganic phase. It is then followed by heating up to $800^{\circ} \mathrm{C}$ at $5^{\circ} \mathrm{C} / \mathrm{min}$ and holding for $1 \mathrm{~h}$. Then, the sample was cooled rapidly to room temperature. Yttria acts as a phase stabilizer, which was expected to prevent zirconia phase change with temperature. For $\mathrm{ZrO} 2$ nanofibers, due to their fabrication process and small grain size, the initial crystalline phase is usually tetragonal. It crystallizes above $350-450{ }^{\circ} \mathrm{C}$ and then slowly converts to monoclinic at higher temperatures. This grown monoclinic phase will still convert to tetragonal above $1170{ }^{\circ} \mathrm{C}$ and then back to monoclinic upon cooling. The conversion to monoclinic during the heating of as-spun nanofibers is prevented by the addition of yttria. So, without yttria, nano fibers would be mostly monoclinic after sintering at $800^{\circ} \mathrm{C}$. But with yttria, they are still fully tetragonal. This is confirmed by $\mathrm{x}$-ray diffraction (XRD) analysis of sintered nanofiber as shown in Fig. 5(c).

\section{RESULTS AND DISCUSSION}

Using the new custom-made high-power supply and dual polarity arrangement of electrospinning setup, it was possible to manufacture thicker mats of ceramic nanofiber with submicron diameter fiber. Figure 4(a) shows fiber mat morphology with out-of-plane point-like structures after long hour of electrospinning without using a negative ionizer. This was most probably due to accumulation of positively charge on the fiber mat. As the electrospinning process proceeds with successive deposition of nanofiber layers, the thickness of mat increases. After it attains certain thickness, the charges on the newer nanofiber layers find it difficult to reach the grounded metal cylinder due to underlying insulating layers of nanofiber. As a result, there is accumulation of surface charge on the nanofiber mat that repels the oncoming positively charged nanofibers and bring the nonuniformity in electrical potential distribution. By employing a negative ionizer, the positively charge nanofibers were immediately neutralized by the negative ions with each rotation of cylindrical collector. Higher rotation speed would align the nanofiber in the circumferential direction and make the fiber mat anisotropic, which was undesirable in our application. Low-rotation speed enabled the formation of nanofiber mat with randomly oriented fibers in a nonwoven form. A cylindrical collector was chosen so that the positively charged nanofibers can be easily neutralized by negative ions from the ionizer in the course of one revolution hence allowing deposition of oncoming fibers without charge repulsion. Figure 4(b) shows absence of such pointed structure and formation of uniform thickness nanofiber mat. The mat 
(a)

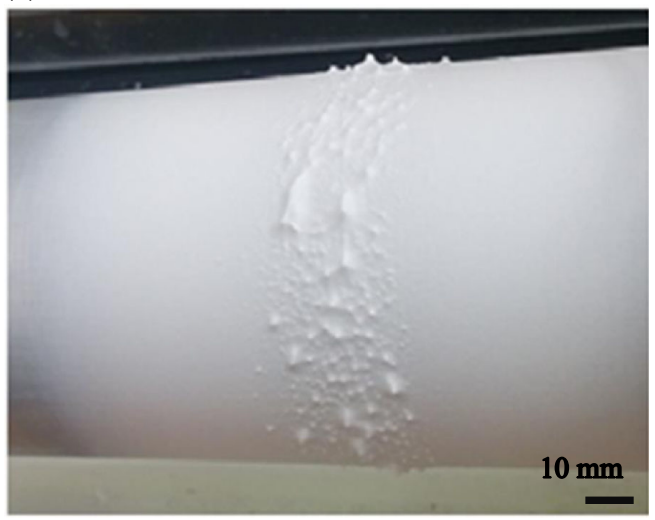

(c)

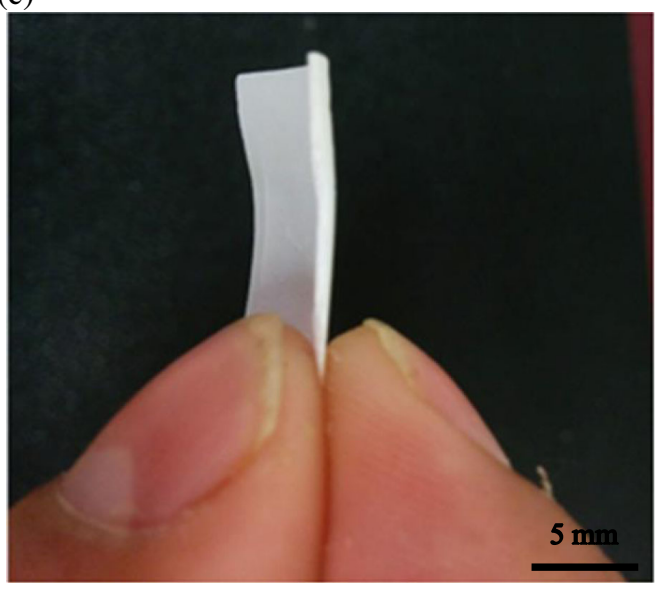

(b)

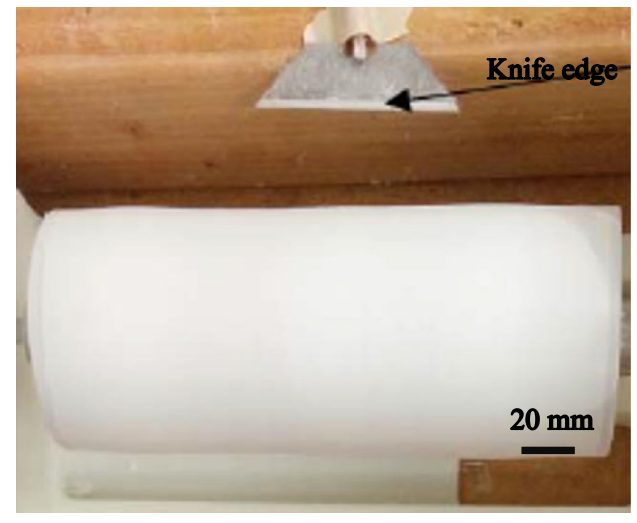

(d)

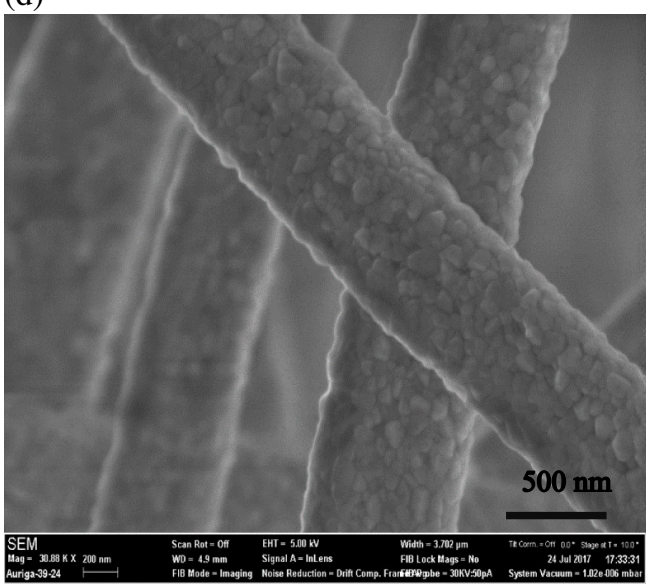

FIG. 4. Thicker Nanofiber mat (a) without negative ionizer (b) with negative ionizer, (c) thick ceramic nanofiber mat after heattreatment, (d) SEM image of zirconia nanofiber revealing polycrystalline grains.

could be easily peeled off from the cylindrical and cut into required size which was then heat treated following the two-stage heating profile described in Fig. 3(b). Figure 4(c) shows one such piece after heat treatment. Figure 4(d) shows a high-resolution scanning electron microscope (SEM) image of single nanofiber with diameter of $500 \mathrm{~nm}$ and revealing polycrystalline grains of $15-50 \mathrm{~nm}$ in size.

\section{A. Microstructure/morphology}

A JOEL-4200 SEM was used to characterize morphology of nanofibers. Figures 5(a)-5(b) show SEM images of nonwoven nanofiber structure in the nanofiber mat before and after heat treatment. The insets in Fig. 5 show energy dispersive $\mathrm{x}$-ray spectroscopy (EDS) mapping of various elements in nanofibers with weight percentage before and after heat treatment. As it can be seen from the weight percentage of constituent elements in Table I, there was drastic reduction of carbon fraction showing absence of any polymer residue in the nanofiber mat after heat treatment. From the weight \% of individual components listed in Table I, it can be estimated that mole\% yttria is around $2.37 \%$. There was reduction in nanofiber mat dimension up to $50 \%$ in all directions. And there was considerable increase in weight $\%$ of zirconium showing formation of ceramic nanofibers in the final product. It can be seen that there was slight deviation from theoretical stochiometric composition of inorganic part and oxygen part in ceramic nanofibers that can be attributed to heat treatment profile. Better composition can be obtained by changing the rate of heating, controlling oxygen flow, and selecting suitable peak temperature and holding time. Also, it is to be noted that the quantitative measurement of light elements like carbon and oxygen is not accurate using EDS and the values mentioned here are for qualitative comparison only. For $\mathrm{ZrO} 2$ nanofibers, due to their fabrication process and small grain size, the initial crystalline phase is usually tetragonal. It crystallizes above $350-450{ }^{\circ} \mathrm{C}$ and then slowly converts to monoclinic at higher temperatures. Note this growth of monoclinic phase will still convert to tetragonal above $1170{ }^{\circ} \mathrm{C}$ and then back to monoclinic upon cooling. The conversion to monoclinic during the heating of as-spun nanofibers is prevented by the addition of yttria. So, without yttria, nano fibers would be mostly monoclinic after sintering at $800^{\circ} \mathrm{C}$. But with yttria, they are still fully tetragonal. Figure 5(c) shows the XRD result 
(a)

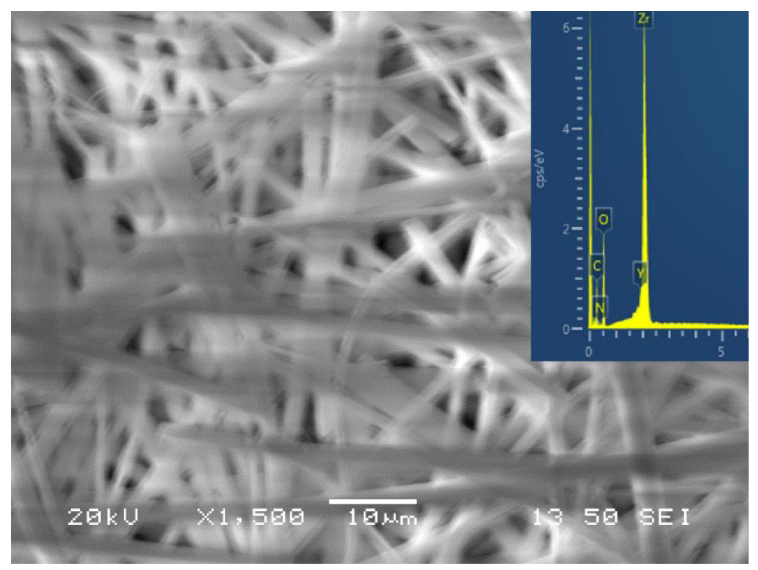

(b)

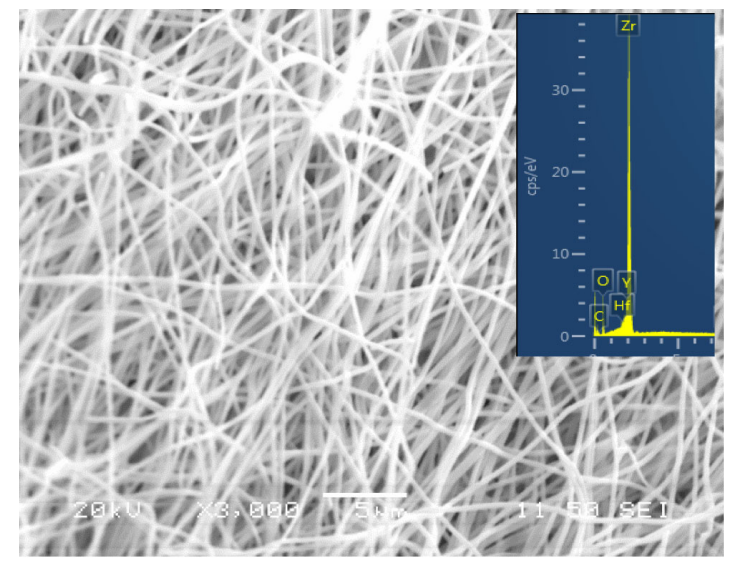

(c)

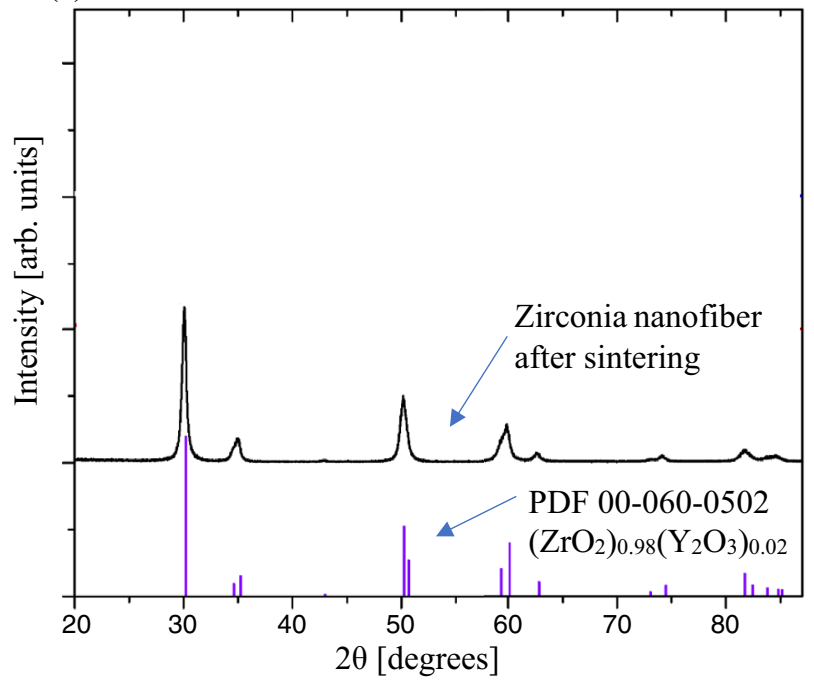

FIG. 5. SEM image of zirconia nanofiber (a) as-spun and (b) after heat treatment. Inserts showing EDS mapping of individual elements. (c) XRD of produced zirconia nanofiber after sintering and reference powder diffraction file of $2 \%$ mole YSZ.

of our sintered nanofiber matches well with reference powder diffraction file of 2 mole \% $\mathrm{Y} 2 \mathrm{O} 3$ in $\mathrm{ZrO} 2$ in tetragonal phase. Hence, the produced zirconia nanofiber was 2 mole \% partial stabilize zirconia.

\section{B. Qualification process}

\section{Micro-mechanical studies on single nanofiber using AFM}

Mechanical property of single nanofiber was carried out using a Bruker icon AFM at Chicago State University. Preparing nanofiber sample for nanoindentation using AFM tip was a major challenge. Individual nanofiber had to be isolated from the bunch of long nonwoven structure of nanofiber mat and fixed on to a substrate material to restrain its movement while probing the nanofiber during the indentation process. Conventional process involves ion milling a piece of nanofiber from the nanofiber mat and fixing it onto a TEM grid using platinum deposition [20-22]. This step is very time-consuming and requires sophisticated equipment like dual-beam focus ion beam /SEM. Some researchers also used a special glue on an ion milled striated substrate to fix a single nanofiber for micromechanical testing [23]. Here, we attempted to achieve similar result using a much easier and inexpensive solution casting method. A chunk of nanofiber plucked using a pair of tweezers and was mixed with $20 \mathrm{ml}$ of deionized water in a beaker. It was then stirred vigorously

TABLE I. Composition of elements in nanofiber mat before and after heat treatment.

\begin{tabular}{lcc}
\hline \hline & Zirconia & \\
\hline Elements & wt\% before & wt\% after \\
\hline $\mathrm{Zr}$ & 31 & 62.43 \\
$\mathrm{C}$ & 26.9 & 5.1 \\
$\mathrm{O}$ & 34.9 & 29.57 \\
$\mathrm{~N}$ & 5.1 & $\ldots$ \\
$\mathrm{Y}$ & 2.2 & 2.9 \\
\hline \hline
\end{tabular}


(a)

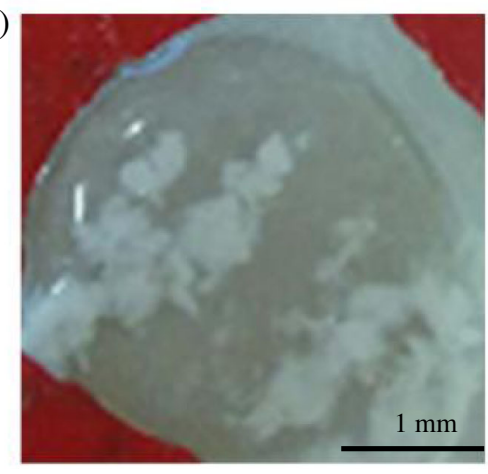

(b)

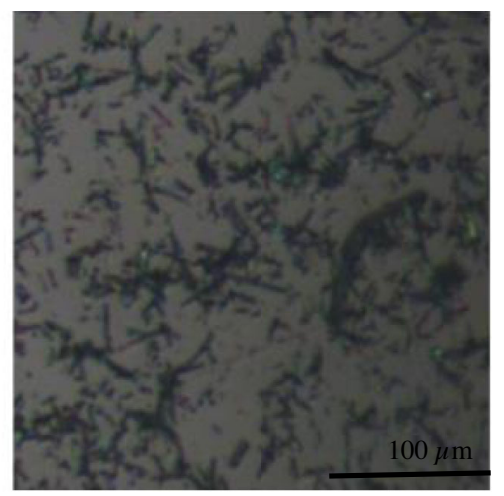

FIG. 6. (a) Solution casting of zirconia nanofiber on mica substrate (b) close-up of dispersed nanofibers on mica substrate after drying.

using a magnetic stirrer in order to break the long nanofibers into smaller fragments and disperse these pieces in the water. Few milliliters of this nanofiber-dispersed solution are taken in a disposable syringe and a few droplets of this was placed on a clean mica disc of $10 \mathrm{~mm}$ diameter [Fig. 6(a)]. It was then left to dry in air for about $6 \mathrm{~h}$ till all the water evaporated [Fig. 6(b)]. Due to nanoscale size of the dispersed nanofiber, it was expected that the fibers would be attached to extremely polished mica substrate by electrostatic force which will be strong enough to resist the indentation force, which is typically in the order of few micro-Newtons for nanoindentation on hard materials.

A diamond tip probe was used to ensure nanometer scale indents with low noise and without tip wearing as it is expected to have a high modulus of elasticity for the ceramic nanofiber. The tip was mounted on a steel cantilever of $300 \mu \mathrm{m}$ long having a nominal resonance frequency of $70 \mathrm{kHz}$ and high-spring constant of $450 \mathrm{~N} / \mathrm{m}$. A stiffer cantilever was used in order to minimize the ringing effect during probe-sample separation. The tip radius was $35 \mathrm{~nm}$ with tip half angle of $18^{\circ}$. The mica substrate with dispersed nanofibers was brought under the optical microscope attached to AFM stage to identify a single isolated nanofiber. Then a square area with a $2 \mu \mathrm{m}$ side was selected around this isolated nanofiber for modulus mapping in order to get large amount of modulus data. To extract mechanical properties, Bruker's Peak force QNM module [24] was used in a tapping mode. In this method, the probe was vibrated at a frequency of $2 \mathrm{kHz}$, much less than the resonance frequency of the cantilever, while it was scanned across the area of interest. The tip only contacts the sample surface for $0.5 \mathrm{~ms}$, keeping the tapping force low and imparting negligible lateral force. Sample deformation depths were limited to few nanometers minimizing loss of resolution due to larger tip-sample contact area. A maximum force of $15 \mu \mathrm{N}$ was chosen in the peak force tapping to protect tip-sample damage while maintaining the contact area to a minimum. A total of $512 \times 512$ points was probed for modulus mapping in $2 \mu \mathrm{m}$ square. At each point, the probe was brought in contact with the sample surface until the force reaches the previously set value of $15 \mu \mathrm{N}$. To separate the contributions from different material properties such as adhesion, modulus, dissipation, and deformation, it was necessary to measure the instantaneous force rather than time averaged force. The force measurement bandwidth of the probe cantilever was approximately equal to the resonant frequency of fundamental bending mode, which enables it to respond to changes in instantaneous interaction force with an immediate deflection change during the peak force tapping. Probe-sample separation was calculated from the cantilever deflection and plotted against the instantaneous force to get force-distance plot that forms the basis of mechanical properties evaluation. Figure 7(a) shows one such plot where forces are plotted during the approach and retraction of the probe. Young's modulus was evaluated from the retraction curve using the Derjaguin-Muller-Toporov (DMT) model [25,26],

$$
F-F_{\mathrm{adh}}=\frac{4}{3} E^{*} \sqrt{R\left(d-d_{0}\right)^{3}},
$$

where $F_{\text {adh }}$ was the adhesive force, $F-F_{\text {adh }}$ was the force on the cantilever relative to adhesion force, $\mathrm{R}$ was the tip radius, and $d-d_{0}$ was the deformation of the sample. $E^{*}$ was the reduced Young's modulus which was related to sample Young's modulus $\left(E_{s}\right)$ through following equation,

$$
E^{*}=\frac{1-\vartheta_{s}^{2}}{E_{s}}+\frac{1-\vartheta_{\mathrm{tip}}^{2}}{E_{\mathrm{tip}}},
$$

where $\vartheta_{s}$ and $\vartheta_{\text {tip }}$ are the Poisson's ratio of sample and indenter tip, respectively. $E_{\text {tip }}$ was assumed to be infinite and sample Poisson's ratio to be 0.22 . The brighter area corresponding to zirconia nanofiber. The contrast in the area mapping was due to probing of cylindrical surface of nanofiber while the darker area corresponds to absence of fiber. It was possible to obtain an image of nanofiber structure based on modulus mapping. Figure 7(c) shows plot of Young's modulus values along a line scan across the 


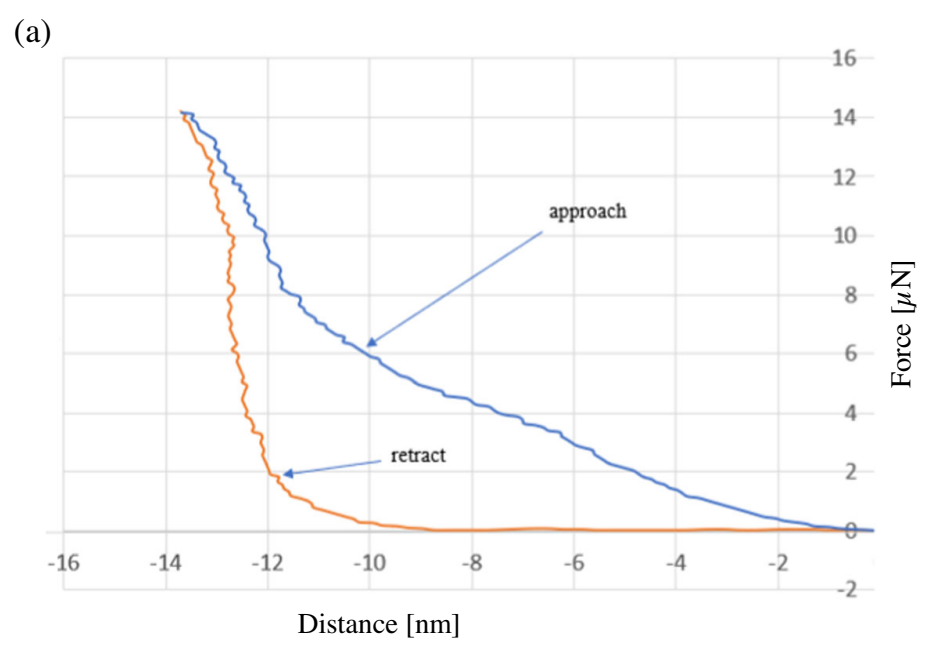

(b)
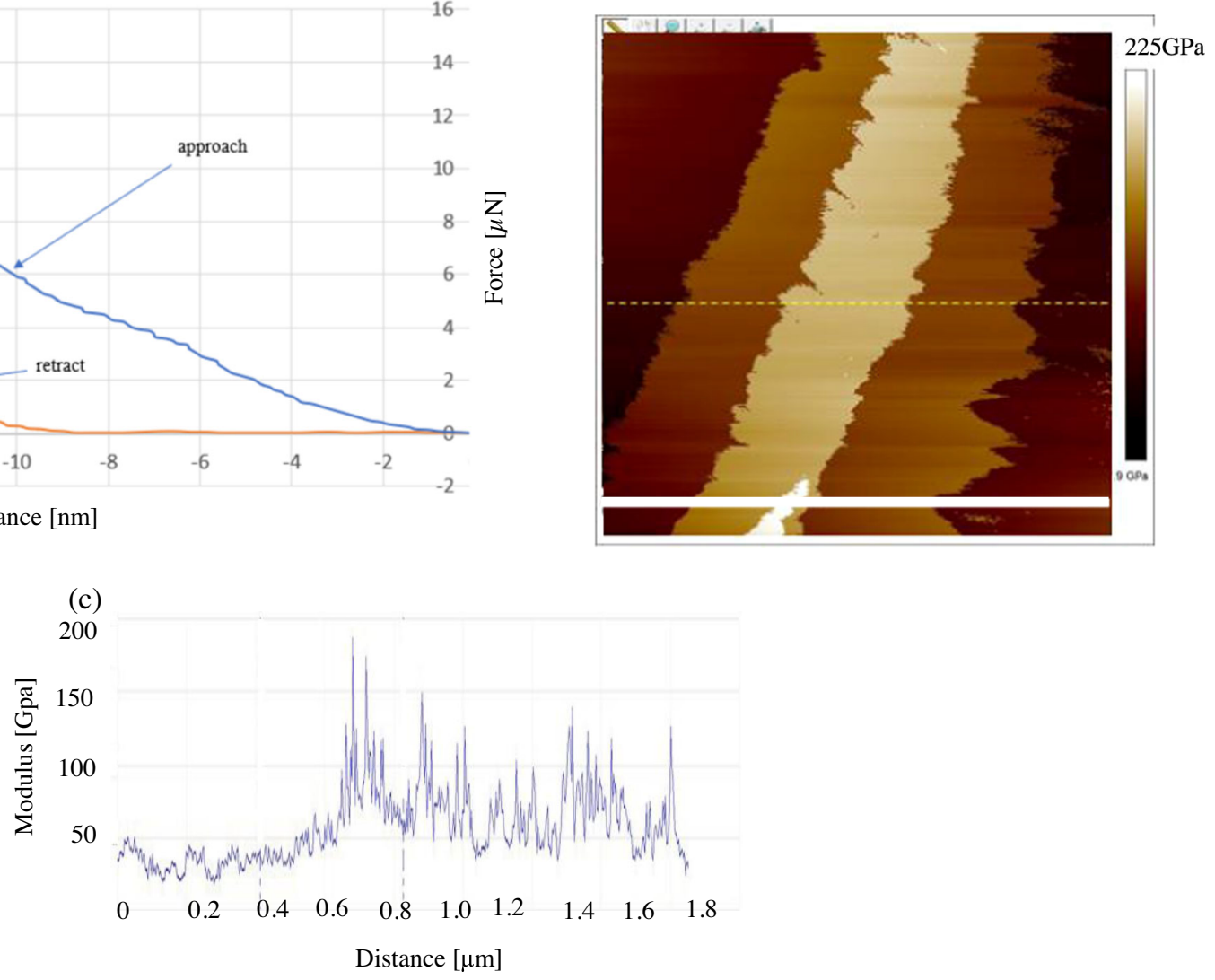

FIG. 7. AFM Micro-mechanical testing on single zirconia nanofiber. (a) Force-distance curve on zirconia nanofiber over one tapping cycle, (b) modulus mapping over a $2 \mu \mathrm{m}$ square containing nanofiber, and (c) Modulus values along a line across the nanofiber.

nanofiber. There was a sharp increase in modulus values along this line where it encounters the nanofiber over a length of about $500 \mathrm{~nm}$. This corresponds to diameter of the nanofiber as previously seen from SEM image in Fig. 4(d). Due to curvature of the nanofiber surface, it was not possible to make a perpendicular contact of nanofiber surface by the AFM tip. The areas where perpendicular point of contact could be made were the points on the nanofiber surface at the highest elevation. Those were found on the line right along the nanofiber axis. Since the exact location of nanofiber surface was not known, an area mapping was conducted around a single nanofiber over a $2 \mu \mathrm{m} \times 2 \mu \mathrm{m}$ area with 512 indentations in horizontal and 512 in vertical axis, a total of $512 \times 512=262144$ indentation points. Figure 7(b) shows modulus mapping over this area. The bright pixels in Fig. 7(b) are the areas of perpendicular contact with AFM tip and reveal actual modulus of the material. These brighter pixels have the value close to $190 \mathrm{GPa}$ as shown in legend bar. There are 512 such bright pixel as there are 512 horizontal indentation scans performed. Hence, we have a lot of data to confirm the modulus to be at least $190 \mathrm{GPa}$. Other areas with lower pixel intensities reflect lower modulus as the contact with AFM tip in these areas were not perpendicular.
The values decrease as the angle of contact decreases and these values were not the true modulus values of the material. The distribution of modulus plotted in Fig. 7(c) is a set of data along a line shown in Fig. 7(b), corresponding to one horizontal scan consisting of 512 indentation points. There will be just one point along this line which corresponds to the highest elevation of nanofiber surface and hence the true modulus of the material which appears as a spike in the line plot of Fig. 7(c) and its value is about $190 \mathrm{GPa}$. The noise along the line corresponds to inclined point of contact with the surface of nanofiber or loose contact with the substrate surface away from nanofiber.

\section{Ion irradiation studies/radiation resistant}

In order to evaluate the resistance against radiation damage, the zirconia few isolated nanofibers were irradiated with low-energy heavy ion at Argonne National Laboratory IVEM Tandem facility [27]. This facility has an in situ TEM attached to ion beam line, which gives a unique opportunity to image defect formation and evolution during irradiation. For in situ TEM purpose, thick layers of nanofiber mat could not be used. Fortunately, no special sample preparation was needed as the zirconia nanofiber diameters were around $100 \mathrm{~nm}$, thin enough for 


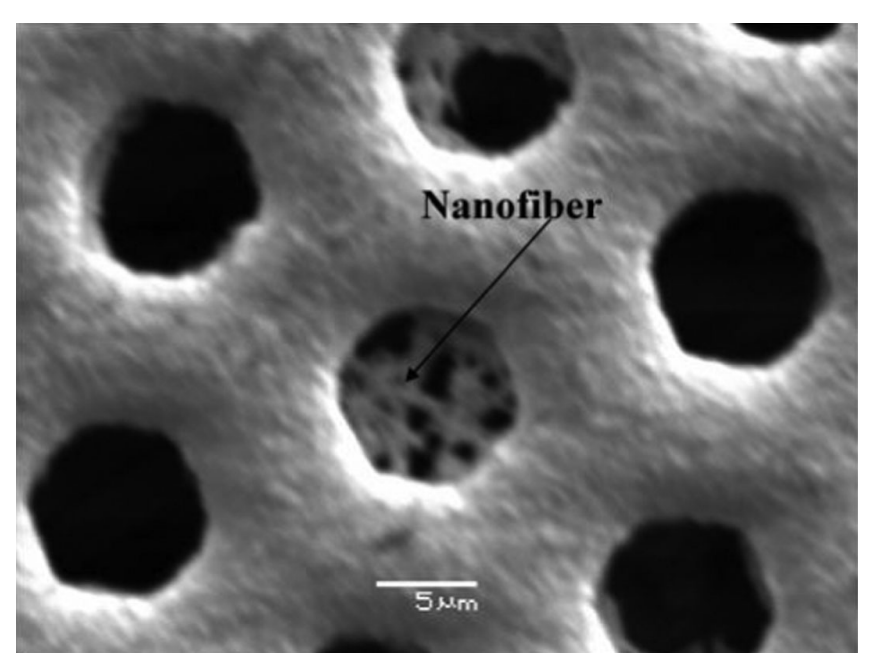

FIG. 8. Solution casted nanofiber on TEM grid.

TEM observation. However, mounting of nanofibers on a substrate for TEM observation was a challenge. Solution casting technique, similar to that used in AFM sample preparation, was adopted for sample mounting. Deionized water solution of dispersed nanofiber fragments was solution casted on a $3 \mathrm{~mm}$ diameter TEM disc with $10 \mu \mathrm{m}$ grid spacing. Figure 8 shows SEM image of a few nanofibers that are hanging over the grid holes. Electrostatic force between the grid surface and nanofibers was strong enough to restrict their movement during ion irradiation and TEM imaging.

$1 \mathrm{MeV} \mathrm{Kr}{ }^{2++}$ ion was used to irradiate the sample by rastering over a circular area of $1.5 \mathrm{~mm}$ diameter with a fluence of $3.25 \times 10^{15} \mathrm{ions} / \mathrm{cm}^{2}$, over a duration of $5000 \mathrm{~s}$. The approximate length of single nanofiber was roughly equal to the TEM grid mesh size which was $10 \mu \mathrm{m}$ and the average diameter of nanofiber was $0.1 \mu \mathrm{m}$. Hence, the actual number of ions received by a single nanofiber on TEM grid hole was $3.25 \times 10^{6}$ ions. The density of single nanofiber is expected to be that of bulk zirconia which is $5.68 \mathrm{~g} / \mathrm{cm}^{3}$. For estimation of energy deposition, MARS simulation was carried out. Due to limitation on geometry in MARS, a single nanofiber could not be simulated. Hence on a $1 \mu \mathrm{m}$ thin zirconia solid plate was used in MARS simulation. Figure 9(a) shows distribution of power density in the thickness direction. From this figure it can be seen that all the energy would have been deposited within a thickness of $150 \mathrm{~nm}$. The average power density in thin plate of zirconia was estimated to be approximately $6670 \mathrm{~W} / \mathrm{cm}^{3}$. For estimating energy transferred to nanofiber, the fraction of ions received on single nanofiber and thickness of nanofiber should be considered. This would translate to a power density of $18.8 \mathrm{~J} / \mathrm{cm}^{3}$ on nanofiber. Assuming density of single isolated nanofiber to be similar to that of bulk zirconia, the expected temperature rise in single nanofiber is calculated to be $2.17{ }^{\circ} \mathrm{C}$ above the room temperature. Under this experiment conditions bulk zirconia would have undergone an average dislocation damage of about 5 dpa as can be seen from Fig. 9(b). Dislocation damage was calculated in SRIM by Kinchin-Pease model. The total vacancies by ions and recoils are added which is further multiplied by atomic density and fluence to get dpa $/ \mathrm{cm}^{2}$.

Figure 10 shows high-resolution bright field and dark field TEM images of zirconia nanofiber before and after irradiation. The dark field images were obtained with small objective aperture selecting the partial of the first two rings of diffraction patterns. Figure 11 shows the selected area diffraction pattern (SADP) before and after irradiation. No new peaks can be evidently observed after irradiation, which indicate the phase stability of the zirconia nanofiber under irradiation. To get a better estimate of lattice parameter from these SADP images,
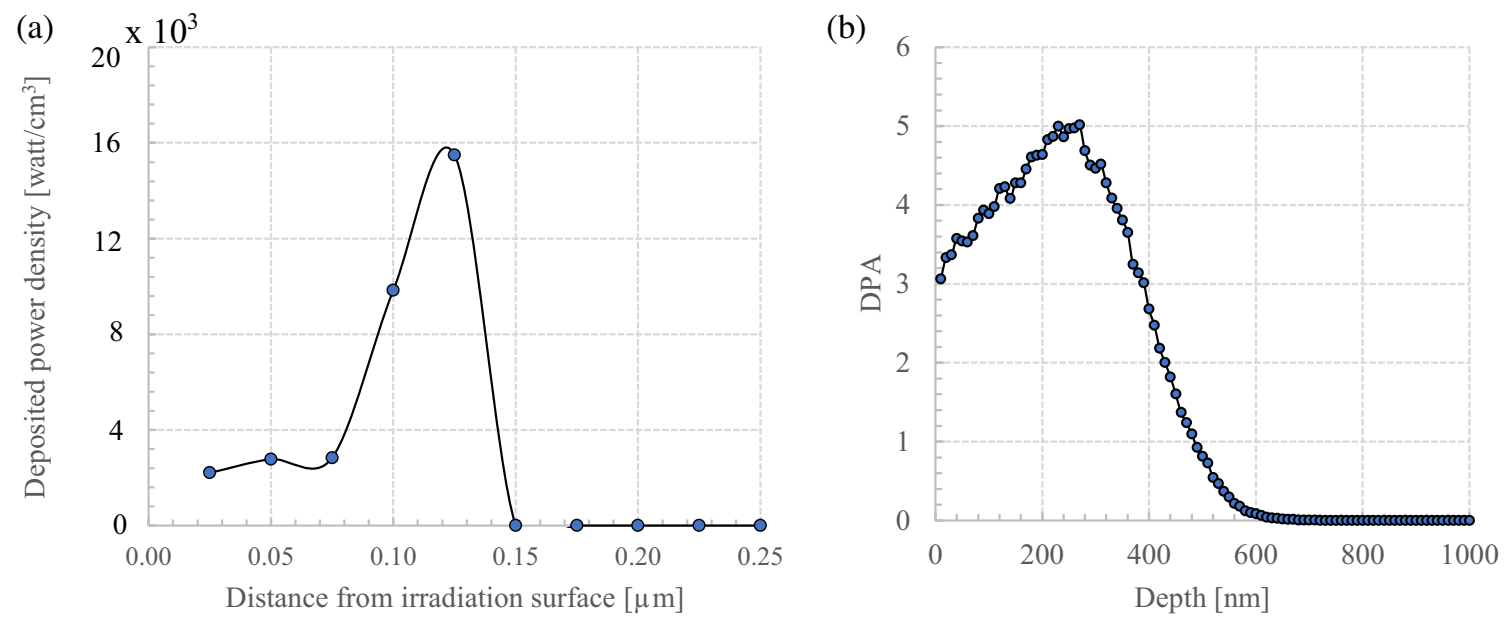

FIG. 9. Simulation result for solid zirconia plate under $1 \mathrm{MeV} \mathrm{Kr}^{++}$ion irradiation. (a) MARS power density and (b) SRIM dpa distribution along thickness direction from the surface of irradiation. 
(a)

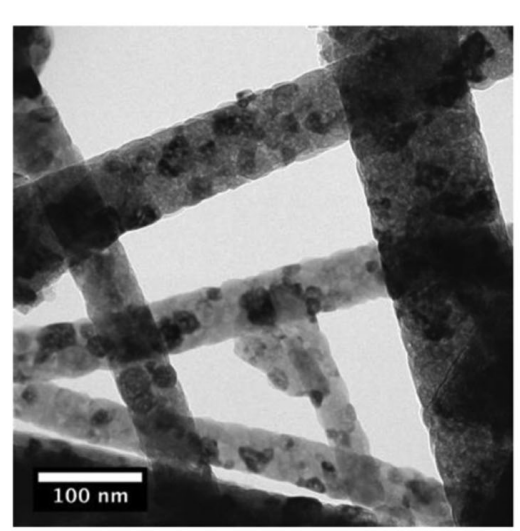

(d)

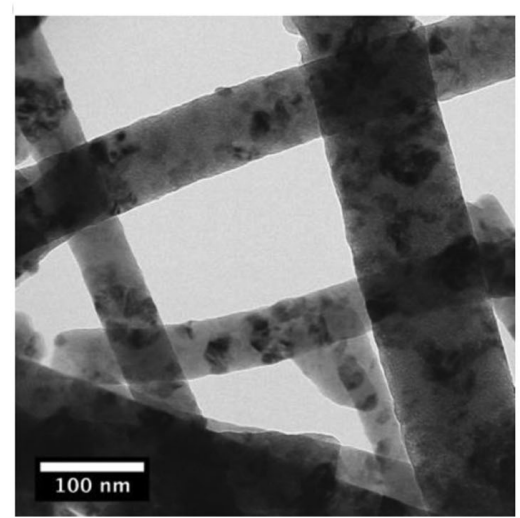

(b)

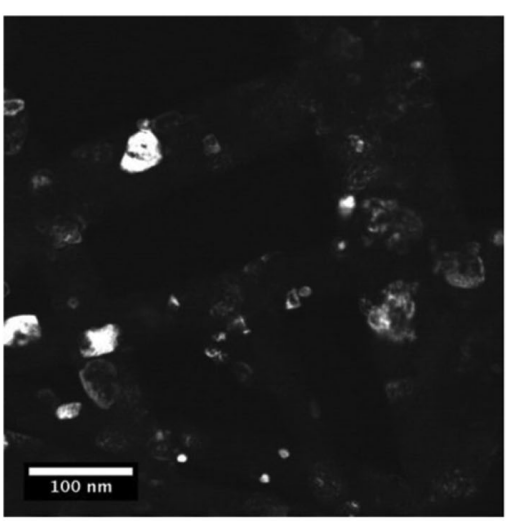

(e)



(c)

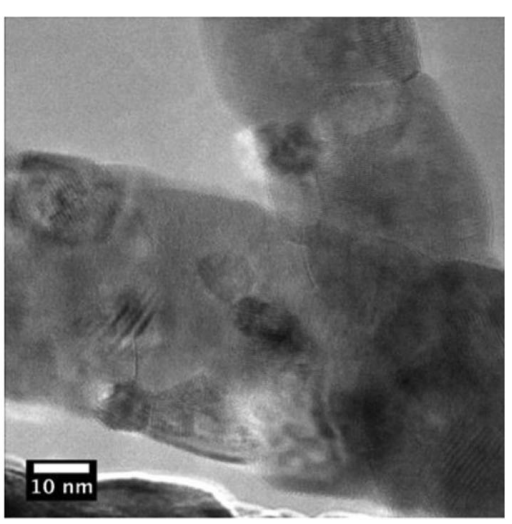

(f)

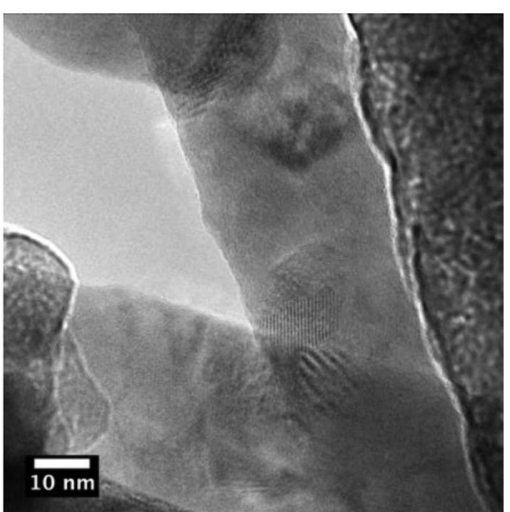

FIG. 10. TEM image of zirconia nanofiber, (a)-(c) before and (d)-(f) after $1 \mathrm{MeV} \mathrm{Kr}^{2++}, 3.1 \times 10^{19}$ ions $/ \mathrm{m}^{2}$. (a) and (d) bright field image. (b) dark field image. (c) and (f) high-resolution images.

a normalized-integrated pixel intensities around concentric circles as a function of distance are plotted using ImageJ [28]. These plots represent inverse $d$-spacing with peak corresponding to various crystallographic planes. Figure 11 shows qualitative comparison of the inverse $d$-spacing plots and hence the lattice parameters before and after ion irradiation. The figures show no measurable changes in peak locations, i.e., lattice parameter or their shapes. The diffraction pattern also indicates that the nanofiber was not amorphized after irradiation. It is worth mentioning that the $d$-spacing values obtained from SADP images are not accurate. More accurate values should be evaluated by XRD method. However, in our case since we are more interested in knowing the differences in lattice parameter due to irradiation rather than evaluating the exact values, SADP inverse $d$-spacing plots are reasonable.

The nano-scale grains of zirconia nanofibers seem to have better resistance to radiation damage due to presence of large number of grain boundaries and high surface areas that would act as a sink to dislocation movements, avoid formation of large voids or dislocation loops. It is anticipated that these closely packed grain boundaries and nanoscale diameter of nanofibers would offer a much smaller path for trapped helium gas formed during proton beam interactions to escape to free surface thereby avoiding swelling.

However, there are some differences in radiation damage caused by high-energy proton beam and low-energy ion beam. Formation of helium gas by transmutation are absent in low-energy ion irradiation. We have chosen ion irradiation over proton irradiation to avoid material activation and to avoid complex and time-consuming procedure of handling activated samples, requirement dedicated expensive equipment for post irradiation examination works. Nonetheless, from TEM image in Fig. 10 it can be seen that ion irradiation did not cause any noticeable dislocations in terms of vacancy or cationic clusters. S. Dey et al., conducted similar irradiation experiment on nano-grain size YSZ where they had demonstrated increase in radiation resistance as the grain size decreases [29]. They reported the sample with $25 \mathrm{~nm}$ grain size showed no difference in XRD pattern before and after irradiation. The grain sizes in our nanofiber material are on the order of 10-15 nm and showed no difference in $d$-spacing response. The TEM images in Dey's et al., work do reveal vacancy and cationic clusters that are not so prominent in our sample. The graphite targets and beryllium window materials in most accelerator environment undergoes less than 1 dpa during their service life. With such low-dpa, we 
(a)

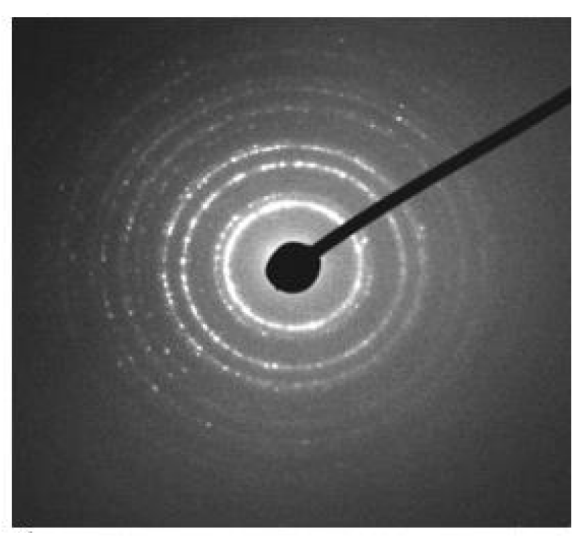

(b)

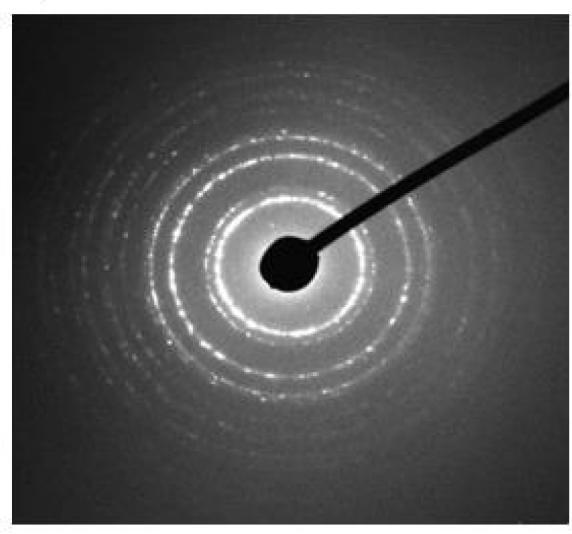

(c) 1.2

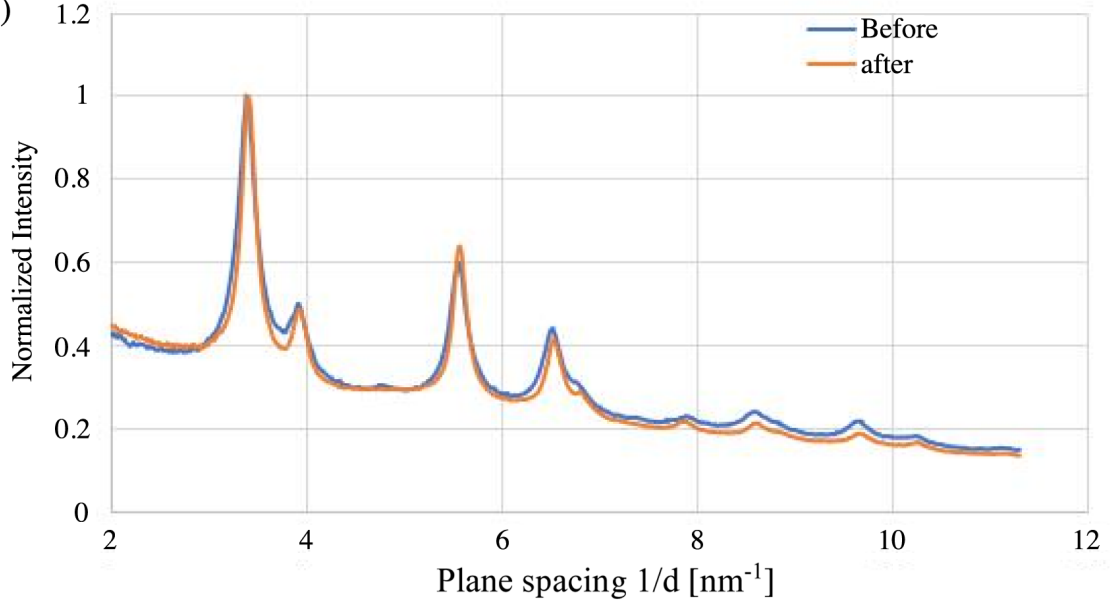

FIG. 11. Selective area diffraction pattern (SADP) image of Zirconia nanofiber before and after $\mathrm{Kr}^{++}$ion irradiation, (c) Comparison of inverse-d spacing plots before and after ion irradiation.

observed peak broadening, reduction in peak height and peak shift of XRD pattern showing deformation to crystal lattice and loss of crystallinity [30]. In our nanofiber sample, we did not observe difference in peak height and width [Fig. 11(c)]. This leads us to believe that our zirconia nanofiber mat may be indifferent to displacement damage up to $5 \mathrm{dpa}$. However, more systematic studies are needed to be done on nanofiber material in order to correlated radiation damage, fluence effects caused by high-energy proton beam and low-energy heavy ion beam. We are currently pursuing this effort too.

\section{Response to electron irradiation}

The purpose of this test was to check the resistance of zirconia nanofibers to temperature rise and induced thermal stresses due to high-energy beam interaction before exposing the samples to high-intensity proton beam of HiRADMat facility. One of the thick zirconia nanofiber mat of $10 \mathrm{~mm} \times$ $10 \mathrm{~mm} \times 0.2 \mathrm{~mm}$ with density of $0.56 \mathrm{~g} / \mathrm{cm}^{3}$ was placed in a plastic holder and irradiated with electron beam at Fermilab's IARC facility [31]. A $9 \mathrm{MeV}$ electron beam with $2.31 \times 10^{12}$ electrons/bunch, bunch width of $2.8 \mu \mathrm{s}$ and
$360 \mathrm{~Hz}$ was used in a continuous mode for $30 \mathrm{~s}$. It has a heating duration of $1 \mathrm{~ms}$ and dwell period of $0.6 \mathrm{~s}$. Beam sigma used was $7.7 \mathrm{~cm}$, much larger than the specimen size for uniform irradiation. This translates to a flux of $8.34 \times$ $10^{14}$ electrons/s on the target. MARS Monte-Carlo simulation was carried out that estimated the peak energy deposition of $0.007 \mathrm{GeV} / \mathrm{cm}^{3} /$ electron on zirconia nanofiber at the beam center with uniform deposition along the fiber. Since the nanofiber mat size was much smaller than beam sigma, a uniform energy deposition over the sample surface was expected. From this, the peak temperature rise during each duty cycle was found to be $361{ }^{\circ} \mathrm{C}$. In Fig. 12(a), it can be seen that the zirconia nanofiber mat held its integrity after electron irradiation. Figure 12(b) shows close-up look at the individual nanofiber that showed no signs of damage, breakage, or local melting. This gives us some confidence to test this material under high-intensity proton beam.

\section{Trial thermal shock response using high-energy proton beam}

After getting encouraging results from electron irradiation, some of the nanofiber samples are exposed to intense 
(a)

(b)

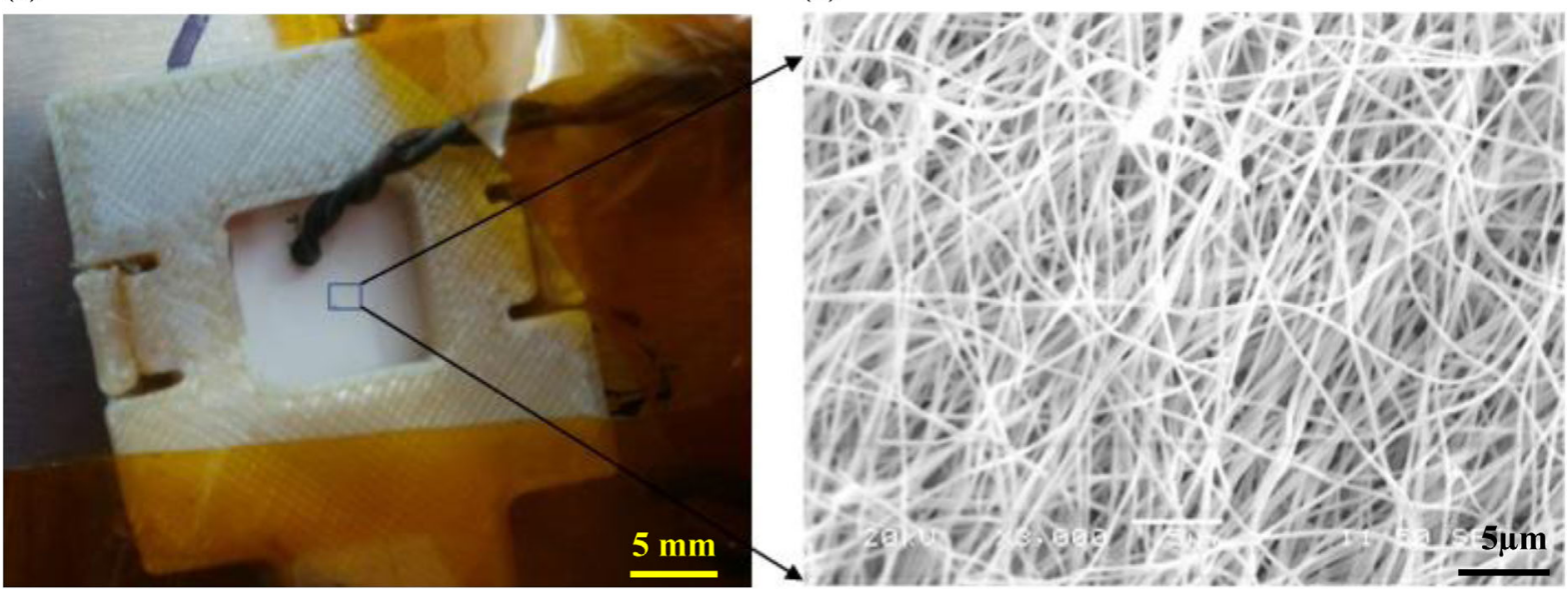

FIG. 12. Zirconia nanofiber mat after electron irradiation (a) inside sample holder and (b) SEM image of nanofibers.

high-energy proton beam for their ultimate qualification as a target material in accelerators. Zirconia nanofiber mats of $10 \mathrm{~mm}$ square and $0.1 \mathrm{~mm}$ thick were placed in a plastic sample holder as shown in Fig. 12(a) and sent to CERN HiRADMat facility [32] for thermal shock tests using a high-energy intense pulsed proton beam. Details of experimental setup are discussed in a previously published paper [33]. It was worth mentioning that there are some differences in sample preparation. Some as-spun nanofiber samples were compressed using a bench vice to increase their packing density and then heat treated to get rid of polymer part. Eight samples were prepared with two different packing densities. Four samples were prepared with density of $1.12 \mathrm{~g} / \mathrm{cm}^{3}$ while other four samples had density of $0.28 \mathrm{~g} / \mathrm{cm}^{3}$. The densities were not accurately measured and estimated by dividing the weight of the sample by the approximate volume of the sample. The error in volume is same for all specimen. There was an uncertainty in length of measurement up to $2 \%$ and in thickness up to $10 \%$. So the error in volume estimation was roughly around $14 \%$. This error in volume estimation is very small in comparison to relative differences in densities of the samples which is four folds in magnitude. Both sets of samples were exposed to single and five pulses separated by approximately $10 \mathrm{~min}$. The environment was normal atmospheric temperature and pressure conditions with no active cooling of samples during irradiation. A $440 \mathrm{GeV}$ pulsed proton beam with Gaussian profile having a beam sigma of $0.25 \mathrm{~mm}$ was used. The pulse duration was $4 \mu \mathrm{s}$ during which $1.21 \times 10^{13}$ protons are bombarded at nanofiber mats. MARS Monte-Carlo simulation was performed to estimate the energy deposition on nanofiber mat. For less densely packed sample the peak energy deposition was estimated to be $0.0712 \mathrm{GeV} / \mathrm{cm}^{3} /$ proton and that of higher density nanofiber mat to be
$0.285 \mathrm{GeV} / \mathrm{cm}^{3} /$ proton. This roughly translates to peak power density of $138 \mathrm{~J} / \mathrm{cm}^{3} /$ pulse on less density sample and $552 \mathrm{~J} / \mathrm{cm}^{3} /$ pulse on high-density sample. Using this information, the instantaneous peak temperature rise is estimated to be $225^{\circ} \mathrm{C}$ in individual fibers located at beam center. Here we assumed physical properties such as specific heat capacity, thermal conductivity of nanofiber to be same as that of bulk yttrium stabilized zirconia. Systematic thermal simulation and characterization of nanofibers should be carried out to estimate real temperature rise in nanofiber mat. Visual inspection of these samples after test shows that the nanofiber mat that were not compacted during fabrication process survived while the samples that were compacted had holes at the beam center. Figures 13(a) and 13(b) show low- and high-magnification SEM images of the loosely packed nanofiber mats that survived the intense pulsed proton beam. Figure 13(c) shows a hole created at the beam center on a densely packed nanofiber sample. The differences in packing density can be easily seen by comparing Figs. 13(b) and 13(d). There was a possibility that some of the carbonized polymer residues are still trapped in the interior layers of nanofiber mat which would have interacted with proton beam to cause additional heat and melting. Also, the densely packed nanofibers in compressed sample would make the heat dissipation difficult from the interior nanofibers at the beam center. However, it was encouraging to see that the other samples have survived the intense proton beam. A more extensive microscopy and SEM image analysis need to be carried out to understand details of the failure modes in damaged samples and state of nanofibers at the beam center in undamaged sample. Systematic studies should be done in future to improve the zirconia content in nanofiber in order to get different phases of zirconia and study their response to proton beam. 
(a)

(b)
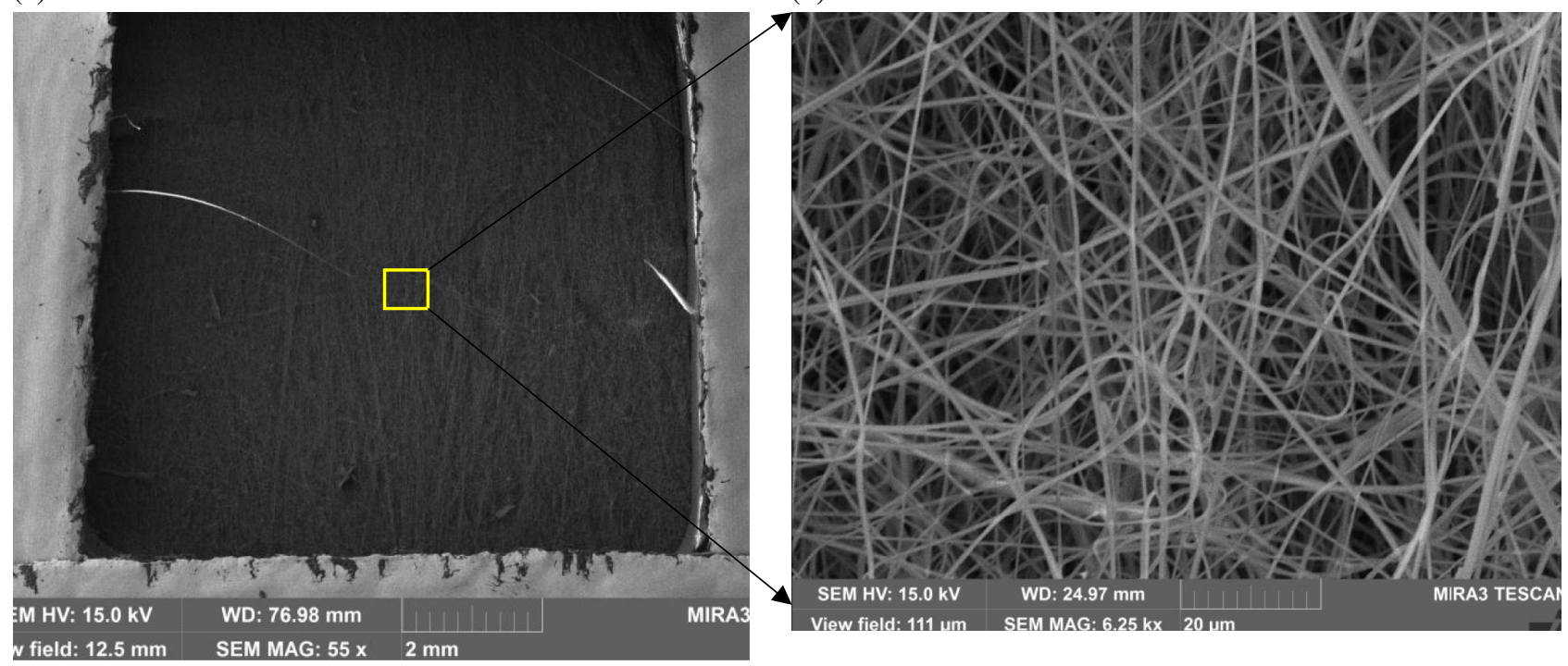

(c)

(d)
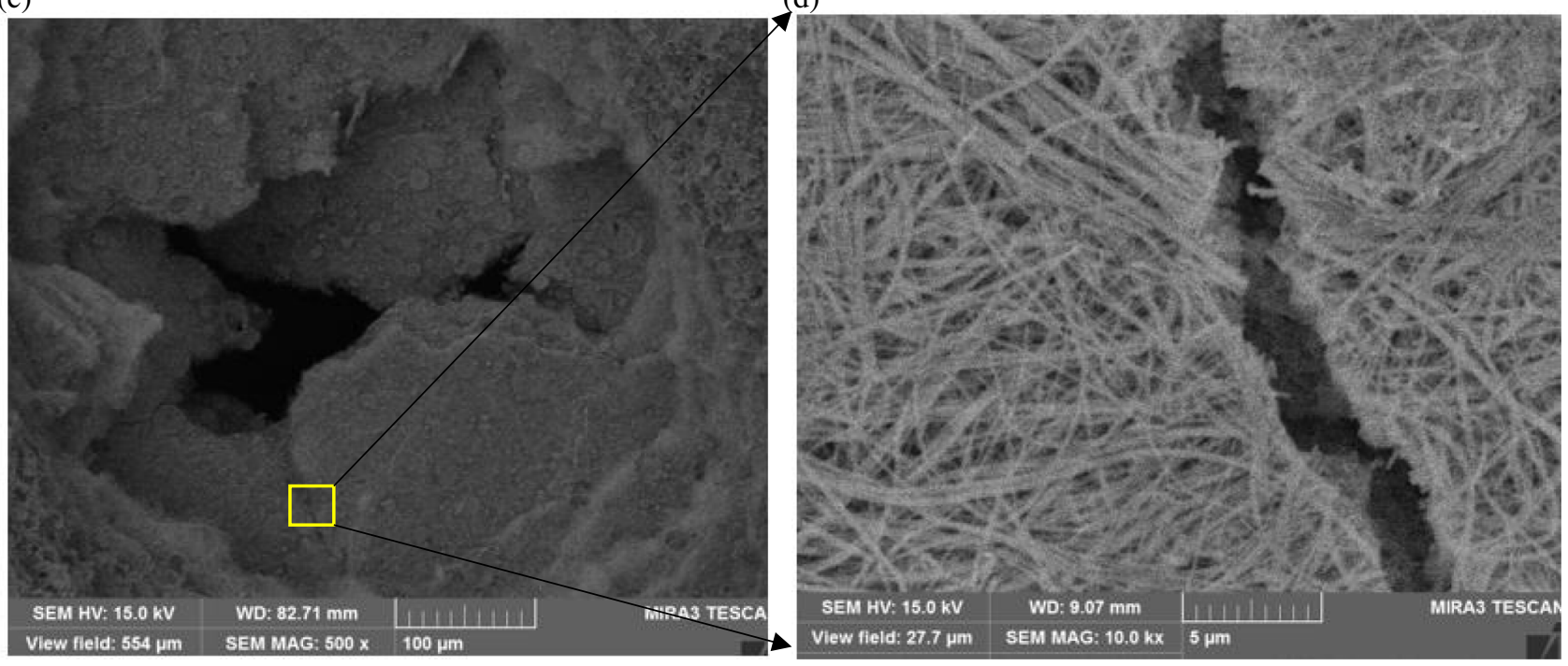

FIG. 13. SEM image of $\mathrm{ZrO} 2$ nanofiber mat after proton irradiation. (a) and (b) loosely packed nanofiber. (c) and (d) densely packed nanofiber with hole at beam center.

\section{CONCLUSIONS}

Zirconia nanofibers of average diameter one hundred nanometers with grain size ranging from 15 to $30 \mathrm{~nm}$ were successfully produced in a thick mat form and tested under intense energetic proton beam as well as low-energy heavy ions and electron beam. A modified electrospinning setup was used to increase the productivity and safety using an inexpensive low-power-output dc-dc high-voltage converter. For the first time ceramic nanofibers were studied under high-energy proton beam to explore a possibility of using such material as targets for neutrino or isotope production. Several innovative techniques were employed in single nanofiber sample preparation for AFM and TEM studies. The produced nanofibers have similar elastic modulus as the bulk zirconia. They did not show noticeable changes in their lattice parameters, grain growth, or new phases under ion irradiation. Nanofibers survived the high-energy electron irradiation in continuous mode that indicates high-temperature resistance. Samples with low-fiber packing density survived the high-energy intense pulsed proton beam, which opens the possibility to use such nanofibers in future highpower targets in high-energy physics research as well as isotope production facilities [34,35]. More material characterization and post irradiation examinations should be carried out to evaluate specific heat, conductance, failure strength, phase, and crystallinity of single nanofiber in order to improve their usability as future targets.

\section{ACKNOWLEDGMENTS}

This manuscript has been authored by Fermi Research Alliance, LLC under Contract No. DE-AC02-07CH11359 with the U.S. Department of Energy, Office of Science, 
Office of High Energy Physics. Financial support was provided under Fermilab LDRD (Laboratory Directed Research and Development) to develop the experimental facility and modified electrospinning setup. We acknowledge the support from NSF Grant No. 1708600 for production of some nanofiber samples. Authors would also like to express special thanks to Fermilab support staff Hiep Le and senior technician Keith Andersen for their contribution in fabricating various components of electrospinning setup. In addition, the European Commission under the FP7 Research Infrastructures project EuCARD-2, Grant Agreement No. 312453, provided support for this experiment.

[1] https://europeanspallationsource.se/target.

[2] https://neutrons.ornl.gov/ppu.

[3] https://news.fnal.gov/2018/04/aiming-at-a-target-thescience-of-particle-production/.

[4] T. Shea, F. Plewinski, A. Nordt, A. Sadeghzadeh, R. Linander, and C. Kharoua, Instrumentation around the ESS $5 \mathrm{MW}$ spallation tungsten target submitted to $2 \mathrm{GeV}$ proton pulses, in Proceedings of 3rd International Conference on Advancements in Nuclear Instrumentation, Measurement Methods and their Applications (ANIMMA), Marseille, 2013 (IEEE Xplore, 2014), pp. 1-8, https:// doi.org/10.1109/ANIMMA.2013.6728029.

[5] O. Caretta, P. Loveridge, J. O’Dell, T. Davenne, M. Fitton, A. Atherton, C. Densham, N. Charitonidis, I. Efthymiopoulos, A. Fabich, M. Guinchard, L. J. Lacny, and B. Lindstrom, Proton beam induced dynamics of tungsten granules, Phys. Rev. Accel. Beams 21, 033401 (2018).

[6] O. Caretta, T. Davenne, C. Densham, M. Fitton, P. Loveridge, J. O'Dell, N. Charitonidis, I. Efthymiopoulos, A. Fabich, and L. Rivkin, Response of a tungsten powder target to an incident high energy proton beam, Phys. Rev. ST Accel. Beams 17, 101005 (2014).

[7] https://web.ornl.gov/info/ridgelines/mercury.htm.

[8] M. Futakawa et al., Cavitation damage prediction for spallation target vessels by assessment of acoustic vibration, J. Nucl. Mater. 377, 182 (2008).

[9] C. J. Ellwason, A. Phatak, D. W. Giles, C. W. Macosko, and F. S. Bates, Melt blown nanofibers: Fiber diameter distributions and onset of fiber breakup, Polymer 48, 3306 (2007).

[10] J. Penide, F. Quintero, J. del Vala, R. Comesaña, F. Lusquiños, A. Riveiroc, and J. Pou, Laser spinning: A new technique for nanofiber production, Phys. Procedia 56, 365 (2014).

[11] J. M. Deitzel, J. D. Kleinmeyer, J. K. Hirvonen, and N. C. B. Tan, Controlled deposition of electrospun poly(ethylene oxide) fibers, Polymer 42, 8163 (2001).

[12] L. Xu, W. Han, G. Zheng, D. Wu, X. Wang, and D. Sun, Initial jet before the onset of effective electrospinning of polymeric nanofibers, Open Mech. Eng. J. 9, 666 (2015).

[13] M. M. Hohman, M. Shin, G. Rutledge, and M. P. Brenner, Electrospinning and electrically forced jets: Stability theory, Phys. Fluids 13, 2201 (2001).
[14] G. Collins, J. Federici, Y. Imura, and L. H. Catalani, Charge generation, charge transport, and residual charge in the electrospinning of polymers: A review of issues and complications, J. Appl. Phys. 111, 044701 (2012).

[15] G. Viswanadam and G. G. Chase, Modified electric fields to control the direction of electrospinning jets, Polymer 54, 1397 (2013).

[16] B. C. Christopher and P. Mckay, Radiation shielding systems using nanotechnology, NASA, U.S. Patent No. 7,923,709, 2011, https://ntrs.nasa.gov/citations/ 20110010999.

[17] Y. Cakmak, M. F. Canbolat, E. Cakmak, and M. Dayik, Production and characterization of boron nitride-doped nanofiber mats created through electrospinning, J. Ind. Text. 47, 993 (2016).

[18] S. Nambiar and J. T. W. Yeow, Polymer-composite materials for radiation protection, ACS Appl. Mater. Interfaces 4, 5717 (2012).

[19] H. Esfahani, R. Jose, and S. Ramakrishna, Electrospun ceramic nanofiber mats today: Synthesis, properties, and applications, Material 10, 1238 (2017).

[20] D. S. Gianola and C. Eberl, Micro- and nanoscale tensile testing of materials, JOM 61, 24 (2009).

[21] E. Zussman, X. Chenb, W. Ding, L. Calabri, D. A. Dikin, J. P. Quintana, and R. S. Ruoff, Mechanical and structural characterization of electrospun PAN-derived carbon nanofibers, Carbon 43, 2175 (2005).

[22] M. Naebe and T. Lin, Carbon Nanotubes Reinforced Electrospun Polymer Nanofibres, Nanofibers (INTECH, Croatia, 2010), pp. 438, ISBN 978-953-7619-86-2.

[23] S. R. Baker, S. Banerjee, K. Bonin, and M. Guthold, Determining the mechanical properties of electrospun poly- $\varepsilon$-caprolactone (PCL) nanofibers using AFM and a novel fiber anchoring technique, Mater. Sci. Eng. C 59, 203 (2016).

[24] https://www.bruker.com/products/surface-and-dimensionalanalysis/atomic-force-microscopes/modes/modes/imagingmodes/peakforce-qnm.html.

[25] D. Maugis, Contact, Adhesion and Rupture of Elastic Solids, (Springer-Verlag, Berlin, 2000), ISBN 978-3-66204125-3.

[26] J. J. Roa, G. Oncins, J. Diaz, F. Sanz, and M. Segarra, Calculation of Young's Modulus Value by Means of AFM, Recent Pat. Nanotechnol. 5, 27 (2011).

[27] https://www.ne.anl.gov/ivem/.

[28] https://imagej.nih.gov/ij/plugins/radial-profile-ext.html.

[29] S. Dey, J. W. Drazin, Y. Wang, J. A. Valdez, T. G. Holesinger, B. P. Uberuaga, and R. H. R. Castro, Radiation tolerance of nanocrystalline ceramics: Insights from Yttria Stabilized Zirconia, Sci. Rep. 5, 7746 (2015).

[30] S. Bidhar, N. Simos, D. Senor, and P. Hurh, Failure investigation of nuclear grade POCO graphite target in high energy neutrino physics through numerical simulation, Nucl. Mater. Energy 4, 100761 (2020).

[31] https://iarc.fnal.gov/capabilities-and-resources/acceleratorapplication-development-and-demonstration/.

[32] https://hiradmat.web.cern.ch/hiradmat-facility.

[33] K. Ammigan et al., Thermal shock experiment of beryllium exposed to intense high energy proton beam pulses, Phys. Rev. Accel. Beams 22, 044501 (2019). 
[34] P. Calvo, I. Podadera, D. Gavela, C. Oliver, A. Adelmann, J. Snuverink, and A. Gsell, Beam stripping interactions in compact cyclotrons, Phys. Rev. Accel. Beams 24, 090101 (2021).

[35] N. Simos, H. Ludewig, H. Kirk, E. Dooryhee, S. Ghose, Z. Zhong, H. Zhong, S. Makimura, K. Yoshimura,
J. R. J. Bennett, G. Kotsinas, Z. Kotsina, and K. T. McDonald, Multi-MW accelerator target material properties under proton irradiation at Brookhaven National Laboratory linear isotope producer, Phys. Rev. Accel. Beams 21, 053001 (2018). 